\title{
Carbon nanotube micropillars trigger guided growth of complex human neural stem cells networks
}

\author{
Gabriela S. Lorite ${ }^{1}(\bowtie)$, Laura Ylä-Outinen ${ }^{2}$, Lauriane Janssen ${ }^{1}$, Olli Pitkänen ${ }^{1}$, Tiina Joki ${ }^{2}$, Janne T. Koivisto ${ }^{3}$, Minna \\ Kellomäki ${ }^{3}$, Robert Vajtai ${ }^{4}$, Susanna Narkilahti ${ }^{2}$, and Krisztian Kordas ${ }^{1}$ \\ ${ }^{1}$ Microelectronics Research Unit, University of Oulu, PO BOX 4500, FI 90014, Finland \\ ${ }^{2}$ NeuroGroup, BioMediTech and Faculty of Medicine and Health technology, Tampere University, Arvo Ylpön katu 34, FI 33520, Finland \\ ${ }^{3}$ Biomaterials \& Tissue Engineering Group, BioMediTech, Faculty of Medicine and Health Technology, Tampere University, PO Box 527, FI 33101, Finland \\ ${ }^{4}$ Department of Materials Science and Nanoengineering, Rice University, P.O. Box 1892, Houston, TX 77251-1892, USA
}

(c) The Author(s) 2019.

Received: 13 May 2019 / Revised: 30 September 2019 / Accepted: 3 October 2019

\begin{abstract}
New strategies for spatially controlled growth of human neurons may provide viable solutions to treat and recover peripheral or spinal cord injuries. While topography cues are known to promote attachment and direct proliferation of many cell types, guided outgrowth of human neurites has been found difficult to achieve so far. Here, three-dimensional (3D) micropatterned carbon nanotube (CNT) templates are used to effectively direct human neurite stem cell growth. By exploiting the mechanical flexibility, electrically conductivity and texture of the 3D CNT micropillars, a perfect environment is created to achieve specific guidance of human neurites, which may lead to enhanced therapeutic effects within the injured spinal cord or peripheral nerves. It is found that the 3D CNT micropillars grant excellent anchoring for adjacent neurites to form seamless neuronal networks that can be grown to any arbitrary shape and size. Apart from clear practical relevance in regenerative medicine, these results using the CNT based templates on Si chips also can pave the road for new types of microelectrode arrays to study cell network electrophysiology.
\end{abstract}

\section{KEYWORDS}

carbon nanotubes, multiple cues, guided neurite outgrowth, human neural stem cells, neuronal networks

The first step of the development of the neuronal network, both in the growing embryo and in vitro, is neurite outgrowth. Through the growth cone, the spreading neurite senses the complex and changing extracellular matrix (ECM) environment thus finding the optimal "road" to establish a functional network [1]. While topography cues are often used as templates in the attempt to guide and orient the neuronal outgrowth as it has been demonstrated using non-conductive polymeric surfaces and two-dimensional (2D) templates, also other environmental cues such as stiffness and biochemistry have been shown to have an impact on cell regulation [2-10]. However, studies providing multiple cues that would combine topography, physicomechanical, electrical and biochemical cues have not yet been explored.

The excellent electrical, mechanical, optical and thermal properties of carbon nanotubes (CNTs) have been exploited in a variety of therapeutic and diagnostic applications $[11,12]$. Recent reports show that CNTs are suitable for adsorbing certain proteins from culture media thus stimulating cell growth, supporting neuron attachment, growth, as well as differentiation and long-term survival [13-16]. CNTs have also been successfully used to mechanically reinforce hydrogels and create electrically conductive nanofibrous networks, enhancing bio-interface with the neural and cardiac cells [17-20]. Furthermore, such electrically conductive cues can also maintain and promote neuronal electrical activity in cultured cell networks, and mimic neural processes when organized into bundles [21, 22]. Recently, it has been reported that ECM conductance is one important electrical cue in neuronal spreading $[23,24]$.
Guided neuron growth for short incubation periods (from $6 \mathrm{~h}$ to 4 days) has been demonstrated with rodent cells on CNT patterns using short nanotubes of $40-500 \mathrm{~nm}$ in length, which lack the microenvironments of the ECM $[15,25]$. Also with rodent neural cells, guided growth was achieved on continuous patterns of $10 \mu \mathrm{m}$-tall aligned CNT forests within a short incubation period

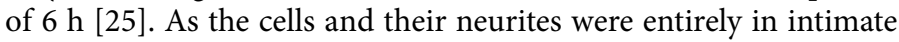
contact with the electrically conductive CNT forest, their functionality must have been compromised because of the formed short circuits. Furthermore, these studies have employed rodents' neural cells, which lack human specific characteristics and thus would require some sort of, yet unknown, extrapolation to mimic and understand human neural cell behavior [26-28].

Here, we report on the application of on-chip three-dimensional (3D) CNT micropillar templates to guide neurite outgrowth resulting in complex neuronal networks with well-defined geometry and long-term cell survival. The neuronal cells grown on our templates prefer to extend the neurites by "hanging" and "wrapping around" the 3D CNT micropillars above the 2D surface and can be guided to grow over long distances of an arbitrary trajectory by simply providing a suitable pattern of segmented CNT pillars. Such control of human neurite outgrowth is anticipated to produce functional connections between neurons thus opening horizons in the development of new strategies for the repair of injured nervous systems.

The above studies inspired us to investigate the control of human neurite outgrowth in a unique environment with electrically conductive 
and mechanically flexible microscopic pillars of CNTs on an insulating and chemically inert surface. Our hypothesis is that tailored 3D CNT micropattern arrays will provide multiple cues including topographical, mechanical, and biochemical factors that are to be considered when mimicking ECM. To investigate our hypotheses, micropillars of highly aligned CNTs organized in different geometries were synthesized by catalytic chemical vapour-phase deposition (CCVD) from acetylene on lithographically defined catalyst micropatterns over $\mathrm{Si} / \mathrm{SiO}_{2}$ chips. Transmission electron microscopy (TEM) and Raman of the synthesized CNT micropillars are shown in Fig. S1 in the Electronic Supplementary Material (ESM). The fabricated templates were sterilized via oxygen plasma treatment, which also makes the CNTs less hydrophobic thus facilitating better cell adhesion and culture media penetration between the pillars. After sterilization, the templates were coated with laminin, which is routinely used as coating material to enhance human neural stem cell (NSC) attachment and neurite outgrowth [5, 7-10]. Finally, human NSCs were plated on the top of the $3 \mathrm{D}$ templates and cultured for prolonged periods of up to 21 days.

Using optimized parameters for the CNT pillar dimensions and spacing, the human neurites can be guided rather well along any created pattern (Fig. 1). As shown in Figs. 1(a)-1(d), a pattern of a spiral in which the micropillars have a size and centre-to-centre spacing of 5 and $10 \mu \mathrm{m}$, respectively, the length of neurites are several millimetres long. In addition to scanning electron micrographs, immunocytochemical staining verified the neuronality of the cells (Fig. 1(d)) showing typical morphology with microtubule associated protein-2 (MAP-2). When the CNT pillars were arranged in three parallel lines forming stripes patterns, the neurites followed and grew along the long stripe (even distances of several millimetres) and had located at a distance of $100 \mu \mathrm{m}$ (Figs. 1(e) and 1(f)). The presence of many dead cells observed by using a Live/Dead ${ }^{\circledR}$ assay (Fig. 1(e)) is reasonable since it is a natural process for developing stem cell derived neuronal cultures. During cell maturation process, some of the cells die while others continue their maturation process. It is not expected that the dead cells would affect the functionality of the live cells. An important result is the fact that most of the dead cells were found outside the pillared CNT patterns suggesting that the CNTs support the long-term survival of human NSCs (Fig. 1(e)).

On the CNT micropillar templates, our results clearly show that the forming neurite network follows the shortest spacing between two pillars (Fig. 1(g)). Indeed, in previous studies using polymeric surfaces, the spacing between features such as pillars and grooves proved to be a critical parameter for the optimal alignment of neurite outgrowth [2]. In excellent agreement with these reports, by using overly large spacing (inter pillar dimension) between the CNT micropillars, we found that the neurons formed large tangled $2 \mathrm{D}$ networks (Fig. S2 in the ESM) similar to those found on smooth surfaces (as shown in the flat templates areas of the images in the panels of Fig. S2(c) in the ESM). Nevertheless, the human neurites were also found to interact with and anchor to the CNT micropillars (the inset of Fig. S2(b) in the ESM).

To assess the particular role of the multifunctional CNTs in the guided neurite outgrowth, reference micropillar arrays of $\mathrm{Si}$ with similar dimensions were used to establish a nearly identical micro-topography environment. While single neurite outgrowth forming highly organized networks can be observed on the $3 \mathrm{D}$ CNT micropillar array (Figs. 2(a) and 3(d)), the formation of tight bundles (fasciculation) is mostly found on the Si micropillar array (Figs. 2(d) and 3(a)). Although the tight bundles are "wrapping" around some of the Si micropillars (Fig. 2(e)), they appear to be more random than guided, i.e. they lack the formation of an organized neurite network. In addition to $\mathrm{Si}$ and CNT micropillar arrays, we also tested cell growth on CNT micropillars coated with polymethylmethacrylate (PMMA) to mimic the poor electrical conductivity of Si pillars while retaining the mechanical flexibility

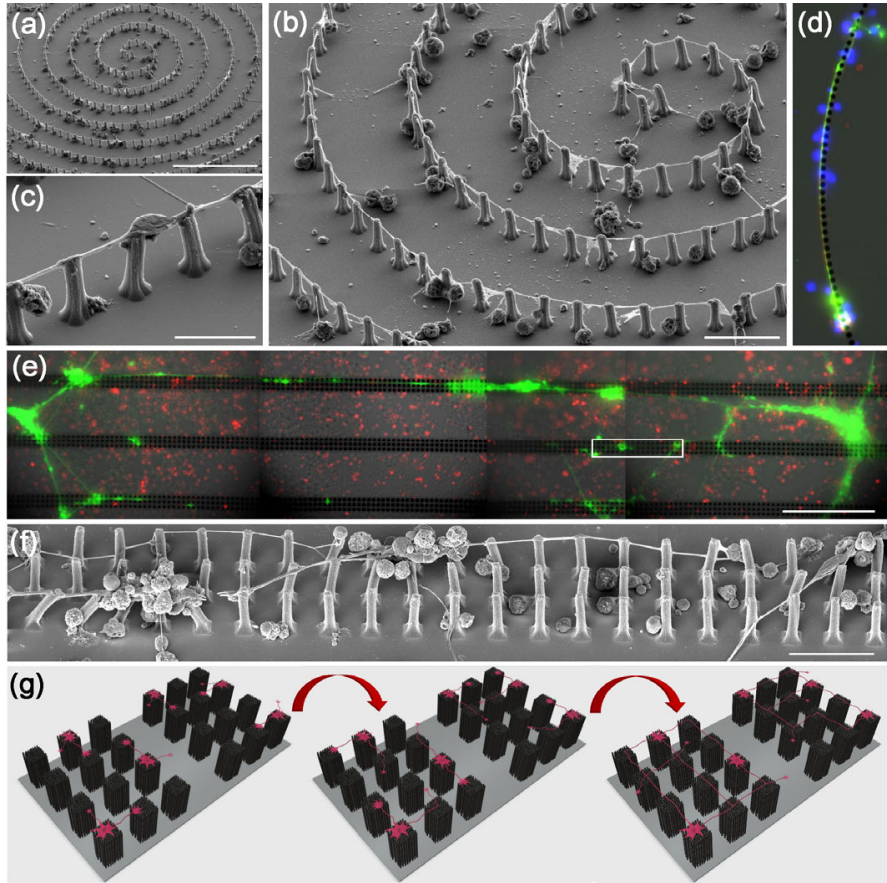

Figure 1 Growth of macroscopic directed networks of human NSCs on CNT micropillar templates with optimized geometries. (a)-(c) Ultra-long, guided neurites stretching along a spiral pattern of several centimetres in total length. Scale bars, $100 \mu \mathrm{m}$ (a), $20 \mu \mathrm{m}$ (b) and $10 \mu \mathrm{m}$ (c). (d) Immunocytochemical staining of a neuron grown on CNT pillars (size of $5 \mu \mathrm{m} \times 5 \mu \mathrm{m}$ with centre-to-centre spacing of $10 \mu \mathrm{m}$ ) along a Fibonacci spiral pattern using neuronal markers MAP-2 (green) for dendrites, neurofilament heavy (red) for axons, and DAPI (blue) for nuclei. (e) Fluorescence microscopy images of Live/Dead ${ }^{\circledR}$ stained human NSCs on parallel stripes of CNT pillars. Green (Calcein-AM) and red (EthD-1) stain intact and dead cells, respectively. Scale bars, $200 \mu \mathrm{m}$. (f) Tilted view (45 ${ }^{\circ}$ SEM images of the guided human NSCs located in the rectangular area highlighted in (e). Scale bar, $20 \mu \mathrm{m}$. (g) Scheme of the human neurite outgrowth strategy showing how the terminals map the surroundings until they find suitable anchoring points, and then proceed further to the next closest location for attachment.

similar to that of the untreated CNT templates (Figs. 3(b) and 3(c)). In these PMMA coated CNT micropillars (pCNT), the middle part of the pattern is not uniformly coated by PMMA (individual CNTs can still be observed from bottom to top of the background pillar (Fig. 3(c))), while the micropillars located on the edge were densely coated by PMMA (Fig. 3(b), background surface). On the densely coated pCNT, the neurite outgrowth is identical to the behavior observed on micropillared Si templates, i.e. tight and thick bundles "wrap" around the micropillars (Figs. 3(a) and 3(b)). On the other hand, a merged behavior of tight bundles "wrapping" around the micropillar and single neurite anchoring on CNTs is found on the partially coated pCNTs (Fig. 3(c)).

Another interesting finding is the physical location of the cells and their neurites on the templates. Instead of being in close contact with the surface of the templates, which is commonly observed on polymeric patterned templates, the human NSCs and their neurites connected the micropillars by "hanging" between them similar to wires between electricity pylons (Figs. 1(c) and 2(a), respectively).

These results can be explained by the additional environmental cues provided by the CNT micropillars. Beyond the microenvironment delivered by the pillars of $10 \mu \mathrm{m}$ in height, the vertically aligned CNTs in each pillar deliver nanotopography cues providing anchoring points with similar dimensions to single human neurites (Figs. 2(b) and 3(d)). Actually, it has been demonstrated that neurons sense and actively respond to nanoroughness with a sensitivity of a few nanometers [20]. Previous studies have shown evidences that the use of CNT films can enhance spontaneous electrophysiological activity of neurons and might affect neural maturity and network 


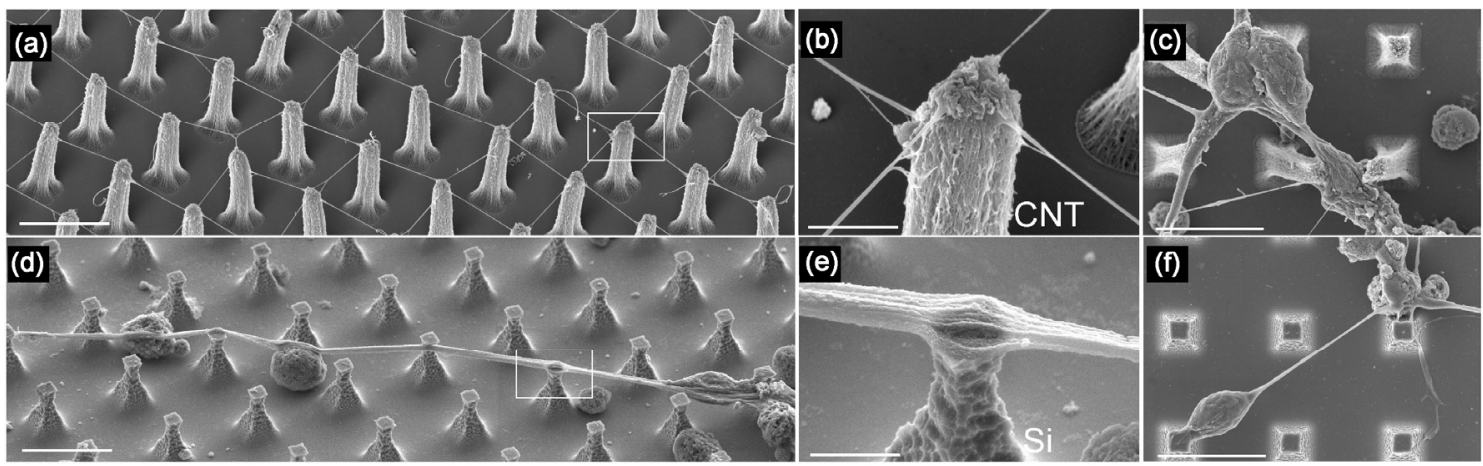

Figure 2 SEM images of human NSCs on 3D micropillared arrays of CNTs and Si. (a) Well-ordered squared pattern network of neurites stretching between the arrayed pillars of CNTs. Scale bar, $5 \mu \mathrm{m}$. (b) Neurites anchored at different locations of the same CNTs pillar. Scale bar, $2 \mu \mathrm{m}$. (c) Human NSCs deforming the mechanically flexible pillars of CNTs. Scale bar, $10 \mu \mathrm{m}$. (d) A single tight bundle of microtubules spanning across Si micropillars. Scale bar, $5 \mu \mathrm{m}$. (e) Tip of a Si pillar wrapped around by the tight bundle of microtubules. Scale bar, $2 \mu \mathrm{m}$. (f) Human NSCs attached to rigid micropillars of Si. Scale bar, $10 \mu \mathrm{m}$.
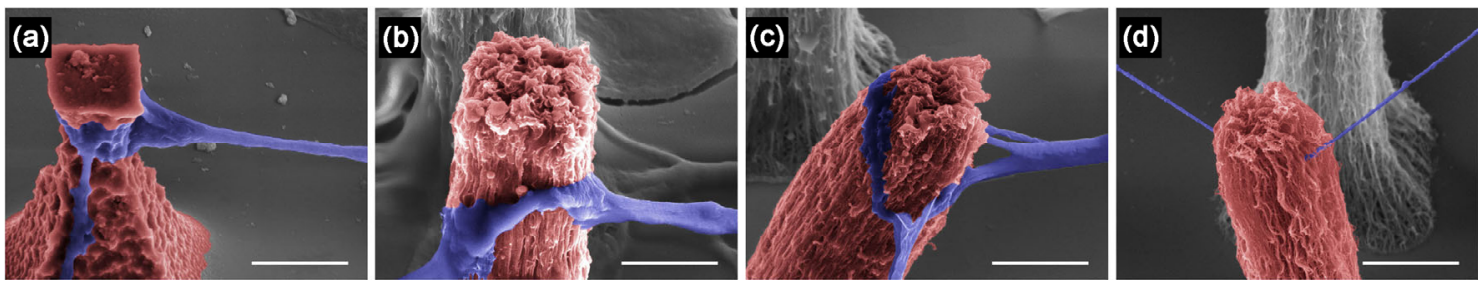

Figure 3 Pseudocolored SEM images of human NSCs on 3D micropillared arrays of Si, pCNT and CNT. (a) Tight and thick bundle of neurite wrapping Si micropillar. (b) Similar neurite behavior is observed on fully coated thus electrically insulating and mechanically stiff pCNT. (c) Tight buddle wrapping the pillar and thinner neurites are observed simultaneously on partially coated pCNT with lower stiffness. (d) Very thin neurite anchoring on CNT micropillars. Original SEM pictures are shown in Fig. S5 in the ESM. Scale bars: $2 \mu \mathrm{m}$.

formation $[21,29]$. Furthermore, we anticipate that the electrically conductive nature of the CNT pillars $(\sim 1 \mathrm{k} \Omega$ between the top and bottom and $\sim 2 \mathrm{k} \Omega$ across the pillar (Fig. S3 in the ESM)) contributes with a further function related to the communication of cells whose neurites are anchored at any point of the same pillar. Although further studies are needed, this hypothesis is also supported by recent publications $[23,24]$.

Another aspect to be considered is the physico-mechanical environment provided by the CNTs in comparison to the Si micropillars (Figs. 2(c) and 2(f)). Previous studies suggest that the mechanical environment provided by the ECM can regulate neuronal cell functions such as signaling, proliferation, differentiation and migration $[6,30]$. In addition, the attachment of neural precursor cells significantly decreases with increased Young's modulus (a 83\% decrease at $35 \mathrm{kPa}$ when compared to $2 \mathrm{kPa}$ ) [31]. The Young's modulus of CNT and pCNT micropillars were estimated using atomic force microscopy (AFM). The Young's modulus for CNT and pCNT micropillars were found in the range of 1.5-5.0 and 75-500 MPa, respectively (Fig. S4 in the ESM). Our findings indicate that the lower Young's modulus provided by the CNT micropillars can facilitate neurite attachment in comparison to stiffer topographies as PCNT and Si micropillars. Furthermore, our scanning electron micrographs indicate that the mechanical flexibility of the CNT pillars helps the cells in their physical accommodation, without being confined between rigid surface features.

The development of neuronal networks relies on the dynamic and complex behavior of the growth cone, which is capable of sensing and translating environmental cues in order to guide the neurite [1]. Although the growth cone mechanism is still far from being completely understood, it is known that the presence of adhesive molecules (e.g. laminin, fibronectin) on the surface with other external chemotropic cues determines the process [1]. We observed the growth cone structure during the initial attachment and proliferation of human NSCs using scanning electron microscopy (SEM). On glass coverslips, the cells developed largely spread growth cones with more finger-like filopodia than on the CNT and Si pillars, in which the growth cone appeared to be more contained (Figs. 4(a)-4(c)). In the latter cases, i.e. on CNT and Si micropillars, the growth cones were found wrapping around both types of pillars (Figs. 4(b) and 4(c)). Similarly as shown for single neurites (Figs. 2(b) and $3(\mathrm{~d}))$, the growth cone structure infiltrates the CNTs micropillars (Fig. 4(c)), indicating that not only the microstructure but also nanoroughness influences the response of the cone receptors.

To assess the response of neurons to the cues of the surface chemistry and laminin, we carried out two parallel experiments with and without laminin coating of the templates. Simple network formations connecting the human NSCs agglomerates to the CNT pillars were observed in both conditions during the initial attachment and proliferation in 1-7 days, which extended to complex human NSCs networks by day 14 (Fig. S6 in the ESM). These results are quite surprising as neurite outgrowth without laminin is known to be rather challenging [7-10]. In fact, we found that even after a single day of incubation on the $3 \mathrm{D}$ micropillared CNT templates coated with laminin, human NSCs were found to form large neurite networks between the pillars (Fig. S7 in the ESM), which survive for at least 14 days (Fig. S6 in the ESM). This result is most probably due to the high protein absorption capacity of the porous and highly specific surface area of the CNT pillars $[13,14]$. Cell experiments on Si micropillar arrays resulted in similar initial attachment and proliferation as on the CNT pillars (1-7 days), but on these templates, complex well-connected networks could not develop when the culturing was continued up to $14-21$ days. It is worth mentioning that without laminin treatment, the neurites could not develop on the Si pillar arrays, which also indicates that the conductive and nanostructured CNT pillars offer a clearly superior cell attachment and growth scaffold for the neurons. The difference is even more striking when looking at the results on smooth glass cover slips, which show no cell growth at all without laminin.

In closing, we demonstrated that tailored 3D CNTs templates provide multiple cues (topographical, mechanical, electrical and chemical) for guided neurite outgrowth and promote the formation of complex neuronal networks of human neural stem cells. The results 

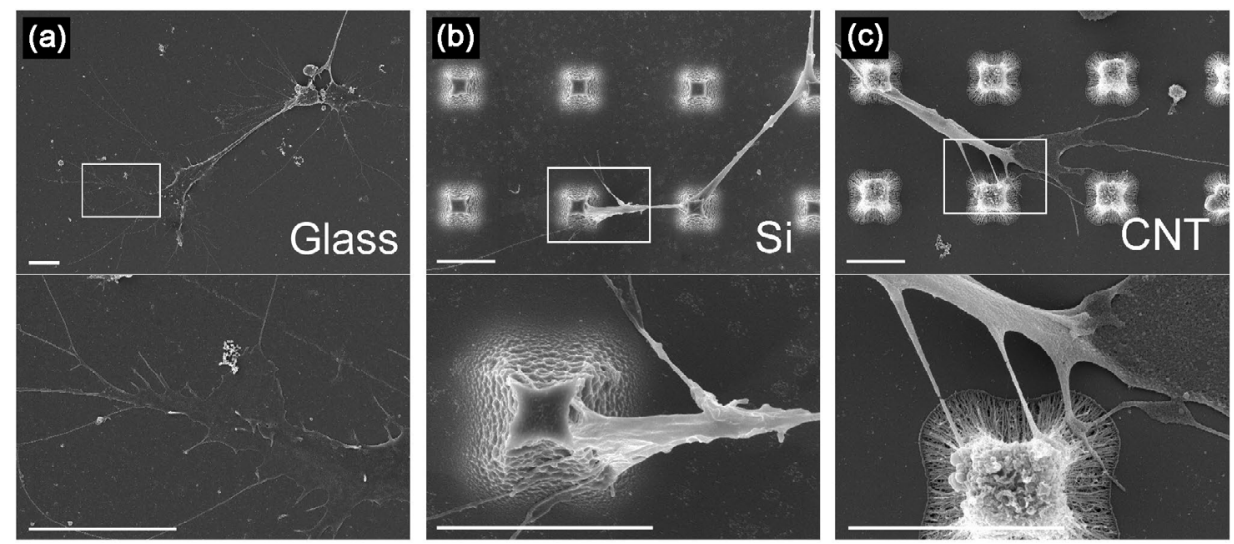

Figure 4 Growth cone propagation and neurite attachment on different template materials. Lower and higher magnification SEM images of a neuron and its growth cone spreading on (a) smooth glass coverslips surface. Scale bars, $10 \mu \mathrm{m}$. Less spread growth cones was observed on (b) Si micropillars and (c) CNT mciropillars. Scale bars, $5 \mu \mathrm{m}$.

indicate that the topology (CNT pillar geometry) directs outgrowth; whereas the mechanically flexible, electrically conductive and nanostructured CNT micropillars provide excellent anchoring points for adjacent (and independent) neurites thus forming seamless neuronal networks that can be grown to any arbitrary shape and size.

\section{Experimental}

Micropillared CNT templates preparation: Multi-walled CNTs were grown on micropatterned templates fabricated using lift-off lithography. First, the photoresist was spin-coated, illuminated and then developed on 4 " Si wafers (Microchemicals $A Z^{\circledR} 1512 \mathrm{HS}$ positive photoresist, Suss RC8 Spinner, Compugraphics 5" Cr-quartz photomask, Suss MA8 Mask Aligner, Microchemicals AZ ${ }^{\circledR} 726$ MIF developer), followed by sputter deposition (Torr International Physical Vapor Deposition System) of an $\mathrm{Al}_{2} \mathrm{O}_{3}$ buffer layer (thickness of $10 \mathrm{~nm}, P_{\mathrm{RF}}=165 \mathrm{~W}, p=2.4 \mathrm{mTorr}$, deposition rate $\left.=0.1 \AA / \mathrm{s}\right)$ and $\mathrm{Fe}$ catalyst (thickness of $\sim 1.5 \mathrm{~nm}, P_{\mathrm{DC}}=30 \mathrm{~W}, p=2.4$ mTorr, deposition rate $=0.1 \AA / \mathrm{s})$. After stripping the photoresist in acetone, the wafer was diced into chips of $9.5 \mathrm{~mm}$ in diameter using a pulsed laser (LPKF ProtoLaser U3, Nd:YVO $, \lambda=355 \mathrm{~nm}, P_{\text {avg }}=6 \mathrm{~W}, f=40 \mathrm{kHz}$, $\tau \sim 20 \mathrm{~ns}$, focal point radius of $\sim 20 \mu \mathrm{m}$ ) and cleaned with acetone and isopropanol. Multi-walled CNTs were synthesized by chemical vapor-phase deposition (CVD) in a cold-walled low-pressure reactor equipped with a 2" heater plate (Aixtron Black Magic). The catalyst was first reduced in $\mathrm{H}_{2}$ (700 sccm, 25 mbar) for 5 min at $700{ }^{\circ} \mathrm{C}$, after which the temperature was lowered to $670{ }^{\circ} \mathrm{C}$ and $\mathrm{C}_{2} \mathrm{H}_{2}$ (20 sccm, $25 \mathrm{mbar}$ ) was introduced to the chamber for $28 \mathrm{~s}$ to grow vertically aligned CNTs of $10 \mu \mathrm{m}$ in height. Various CNT micropatterns were made including (i) squared arrays of pillars (with pillar footprint sizes of $5 \mu \mathrm{m} \times 5 \mu \mathrm{m}$ and $20 \mu \mathrm{m} \times 20 \mu \mathrm{m}$, with centre-to-centre distances of 10, 45, 70 and $220 \mu \mathrm{m}$ ); (ii) pillars along spiral patterns (pillar footprint size of $5 \mu \mathrm{m} \times 5 \mu \mathrm{m}$, with centre-to-centre distances of $10 \mu \mathrm{m}$, and with $20 \mu \mathrm{m}$ as well as $50 \mu \mathrm{m}$ distances between successive turns of the spiral); (iii) pillars arranged in stripes (pillar footprint size of $5 \mu \mathrm{m} \times 5 \mu \mathrm{m}$, with centre-to-centre distances of $10 \mu \mathrm{m}$, and with line spacings of 5-150 $\mu \mathrm{m}$ ) and (iv) arrays of u-shape patterns (both continuous with $5 \mu \mathrm{m}$ width and segmented into pillars of $5 \mu \mathrm{m} \times 5 \mu \mathrm{m}$ in footprint size with $10 \mu \mathrm{m}$ centre-to-centre distances). It must be noted here that the crosssection of the pillars after cell plating is somewhat shrunk due to van der Waals forces that collapse the porous structure. After drying, the cross-section for the $5 \mu \mathrm{m} \times 5 \mu \mathrm{m}$ pillars decreases to $\sim 2.2 \mu \mathrm{m} \times$ $2.2 \mu \mathrm{m}$, whereas for the $20 \mu \mathrm{m} \times 20 \mu \mathrm{m}$ pillars it becomes $13.8 \mu \mathrm{m} \times$ $13.8 \mu \mathrm{m}$.

Micropillared Si templates preparation: Si micropillar arrays (with similar geometries to those made also of CNTs) were patterned on 4" silicon wafers by means of reactive ion etching (RIE, Oxford Instruments Plasmalab 80 Plus, $P=200 \mathrm{~W}, p=70$ mTorr, $6 \mathrm{sccm}$ SF6 flow, $2 \mathrm{sccm} \mathrm{O}_{2}$ flow, temperature $20{ }^{\circ} \mathrm{C}$ with liquid $\mathrm{N}_{2}$ cooling of the sample holder, $40 \mathrm{~min}$ process time) using sputtered and lithographically defined $\mathrm{Cr}$ hard mask patterns (with a thickness of $50 \mathrm{~nm}$ ). After the Si etching process, the $\mathrm{Cr}$ hard mask and natural surface oxides of $\mathrm{Si}$ were removed with subsequent wet etching in aqueous solutions of $9 \% \mathrm{HClO}_{4}$ and $20 \%\left(\mathrm{NH}_{4}\right)_{2} \mathrm{Ce}\left(\mathrm{NO}_{3}\right)_{6}$ as well as in aqueous $\mathrm{HF}(5 \%)$ buffered in $\mathrm{NH}_{4} \mathrm{~F}(36 \%)$. Finally, the wafer was diced into chips of $9.5 \mathrm{~mm}$ in diameter using the same pulsed laser, and then cleaned with acetone and isopropanol.

Passivated CNT micropillars preparation: The CNT templates were immersed in 3 wt.\% PMMA (Degalan LP 50/02, $60 \mathrm{~kg} / \mathrm{mol}$ molecular weight) in toluene solution inside a beaker with a stand and a low speed stirring. After $1 \mathrm{~min}$ the samples were taken out and dried in air overnight.

TEM: CNTs from micropatterned pillared templates were carefully scrapped out and suspended in ethanol. A drop of the suspension was dried at room temperature on $\mathrm{Cu}$ sample holder grids covered with holey carbon (HC300-Cu, Electron Microscopy Science). The TEM images were acquired using a JEOL JEM-2200FS transmission electron microscope. The outer diameter of CNTs was evaluated using EM measure software Beta 0.85 (Teitz Video and Image Processing Systems $\mathrm{GmbH}$ ) by analyzing the distance between the outer dark lines at the edges of the tube walls.

Raman spectroscopy: CNT micropillars were also evaluated with micro-Raman spectroscopy (Horiba Jobin-Yvon LabRAM HR800, $\mathrm{Ar}^{+}$laser source at $\lambda=488 \mathrm{~nm}$ ).

Atomic force microscopy (AFM): Young's modulus of CNT and pCNT pillars were evaluated by using AFM (Bruker Multimode 8), using $10.0 \mu \mathrm{m}$ borosilicate glass sphere probes (Novascan Silicon AFM probe), with Young's modulus $14 \mathrm{~N} / \mathrm{m}$. Each cantilever used in this study was calibrated using touch-calibration of the Bruker NanoScope 9.2 software. Indentation measurements were conducted in air for a threshold of $50 \mathrm{~nm}$ absolute indentation at $3 \mu \mathrm{m} / \mathrm{s}$ with a ramp size of $500 \mathrm{~nm}$. The Young's modulus was extracted from 50-90 micropillars by using the Hertzian (spherical) indentation model. This is obtained by measuring the slope on the initial 30\% of the unloading (retract) load-displacement curve with a linearized model of each ramp. The results are showed as a distribution graph.

Human NSCs culture: The human neurons used in this study were commercial human neural precursor cells hNP1 (ArunA Biomedical, Inc. Athens, GA, USA, LOT: 7003-001, p0), obtained from the human embryonic stem cell (hESC) line WA09. The hNP1 neural precursors were maintained according to the manufacturer's instructions and 
cultured according to the published protocol [32].

Templated cell culture experiments: CNT and Si templates were placed in 24-well plates and sterilized via oxygen plasma treatment for $40 \mathrm{~s}$ with power of $30 \mathrm{~W}$ (Plasma Surface Technology, Electronics Diener). Glass coverslips were used as reference templates. Two parallel experiments were carried out in which human NSCs were plated on the templates (i) right after sterilization as well as (ii) after sterilization and subsequent coating with laminin $(20 \mu \mathrm{m} / \mathrm{mL}$ mouse laminin in phosphate buffered saline (PBS), Sigma Aldrich) for $1 \mathrm{~h}$.

Cell plating: The plating density was $\sim 40,000$ cells/well plate. The cells were cultured on a Neurobasal medium supplemented with $2 \mathrm{mM}$ GlutaMax ${ }^{\mathrm{TM}}, 1 \times$ B27 (all from Gibco, Life Technologies, Finland) and $25 \mathrm{U} / \mathrm{mL}$ penicillin/streptomycin (Cambrex, Belgium). The cells were cultured on the templates for 21 days (at $+37^{\circ} \mathrm{C}$, in $5 \% \mathrm{CO}_{2}$ ). For each analysis timepoint, a set of six samples were taken. During the culture, half of the medium was changed three times per week.

Scanning electron micrographs: The acquisition of electron micrographs was carried out using a field emission scanning electron microscope (FESEM, Zeiss Ultra Plus). Before imaging, the cells were fixed with $5 \%$ glutaraldehyde (Sigma Aldrich) in PBS at room temperature for $1 \mathrm{~h}$. The samples were stored in PBS until imaging. One day before imaging, the samples were dehydrated using ethanol solutions with an ascending series of concentrations (10 vol.\%, $20 \mathrm{vol} \%$, 40 vol.\%, 60 vol.\%, 80 vol.\% and 99.5 vol.\%) for $10 \mathrm{~min}$ each and dried overnight in a vacuum. Finally, the samples were sputter coated with Pt of $\sim 5 \mathrm{~nm}$ (Agar High Resolution Sputter Coated 208HR). Three sets of samples were analysed at each timepoint $(1,2,3,7,14$ and 21 days) of the experiments.

Live/Dead staining: Cell viability was analysed with fluorescencebased Live/Dead ${ }^{\circledR}$ viability/cytotoxicity assay (Thermo Fisher Scientific). The labelling kit contains two labels, Calcein-AM $\left(0.1 \mu \mathrm{M}, \lambda_{\text {excitation }}=488 \mathrm{~nm}\right)$ which stains intact cells and ethidium homodimer-1 $\left(0.4 \mu \mathrm{M}, \lambda_{\text {excitation }}=568 \mathrm{~nm}\right)$ which stains dead cells. The labels were diluted to be suitable for a cell culture medium at the appropriate concentration and incubated for $30 \mathrm{~min}$ at $+37^{\circ} \mathrm{C}$ on the cells. Fluorescence images were acquired with a Nikon FN1 Upright Fluorescence Microscope. Three sets of samples were analysed at each time point (7,14 and 21 days).

Immunostaining: For immunocytochemistry, the cultures were fixed in aqueous paraformaldehyde (4\%) at room temperature for $15 \mathrm{~min}$. After washing in PBS, the cultures were incubated for 45 min with a blocking solution containing 10 vol.\% normal donkey serum (NDS), 0.1 vol.\% Triton X-100, and $1 \%$ bovine serum albumin (BSA) in PBS at room temperature, followed by another wash with $1 \%$ NDS, $0.1 \%$ Triton X-100 and $1 \%$ BSA in PBS. Then, the primary antibodies were incubated in $1 \%$ NDS, $0.1 \%$ Triton X-100, and $1 \%$ BSA in PBS at $+4{ }^{\circ} \mathrm{C}$ overnight on the cells. The antibodies used were: MAP-2 (1:2,000, ab107216, Abcam, UK) and $\beta$-tubulinIII (1:200, NB300-213, Bio-Techne Ltd., UK). After primary antibody incubation, the samples were washed three times for $5 \mathrm{~min}$ in $1 \%$ BSA in PBS. The secondary antibodies (1:400) or TRITC-phalloidin $(0.625 \mu \mathrm{g} / \mathrm{mL}$, Sigma Aldrich, P1951) were incubated with the cells for $1 \mathrm{~h}$ in $1 \%$ BSA in PBS. After secondary antibody incubation, the samples were again washed three times for $5 \mathrm{~min}$ in PBS and left in PBS. DAPI 1:1,000 was included in the first PBS wash. The immunofluorescence images were acquired with a Nikon FN1 Upright Fluorescence Microscope. Three parallel samples were analysed at 7,14 and 21 days.

\section{Acknowledgements}

G. S. L. and L. Y-O. acknowledge the support from the Academy of Finland (Nos. 320090, 317437 and 286990, respectively). J. T. K. and T. J. acknowledge the support from the Finnish Cultural Foundation Pirkanmaa Regional Fund (No. 50151501) and the Central Fund (\#00150312), respectively. S. N., T. J. and M. K. acknowledge the support from the Academy of Finland (S. N. and T. J. No. 312414 and M. K. No. 312409) and Business Finland (former Tekes, Human Spare Parts project). This work made use of the electron microscopy and clean-room facilities at the Centre of Microscopy and Nanotechnology, at the University of Oulu. The authors also acknowledge the Tampere Imaging Facility (TIF) and the Tampere CellTech Laboratories for their service.

Funding: Open access funding provided by University of Oulu including Oulu University Hospital.

Electronic Supplementary Material: Supplementary material (cell attachment and growth of human NSCs on 3D CNT micropillar arrays) is available in the online version of this article at https://doi.org/10.1007/s12274-019-2533-2.

Open Access: This article is licensed under a Creative Commons Attribution 4.0 International License, which permits use, sharing, adaptation, distribution and reproduction in any medium or format, as long as you give appropriate credit to the original author(s) and the source, provide a link to the Creative Commons licence, and indicate if changes were made.

The images or other third party material in this article are included in the article's Creative Commons licence, unless indicated otherwise in a credit line to the material. If material is not included in the article's Creative Commons licence and your intended use is not permitted by statutory regulation or exceeds the permitted use, you will need to obtain permission directly from the copyright holder.

To view a copy of this licence, visit http://creativecommons.org/ licenses/by/4.0/.

\section{References}

[1] Lowery, L. A.; van Vactor, D. The trip of the tip: Understanding the growth cone machinery. Nat. Rev. Mol. Cell Biol. 2009, 10, 332-343.

[2] Simitzi, C.; Ranella, A.; Stratakis, E. Controlling the morphology and outgrowth of nerve and neuroglial cells: The effect of surface topography. Acta Biomater. 2017, 51, 21-52.

[3] Li, W.; Tang, Q. Y.; Jadhav, A. D.; Narang, A.; Qian, W. X.; Shi, P.; Pang, S. W. Large-scale topographical screen for investigation of physical neural-guidance cues. Sci. Rep. 2015, 5, 8644.

[4] Solanki, A.; Chueng, S. T. D.; Yin, P. T.; Kappera, R.; Chhowalla, M.; Lee, K. B. Axonal alignment and enhanced neuronal differentiation of neural stem cells on graphene-nanoparticle hybrid structures. Adv. Mater. 2013, $25,5477-5482$.

[5] Hyysalo, A.; Ristola, M.; Joki, T.; Honkanen, M.; Vippola, M.; Narkilahti, $\mathrm{S}$. Aligned poly( $\varepsilon$-caprolactone) nanofibers guide the orientation and migration of human pluripotent stem cell-derived neurons, astrocytes, and oligodendrocyte precursor cells in vitro. Macromol. Biosci. 2017, 17, 1600517.

[6] Fu, J. P.; Wang, Y. K.; Yang, M. T.; Desai, R. A.; Yu, X.; Liu, Z. J.; Chen, C. S. Mechanical regulation of cell function with geometrically modulated elastomeric substrates. Nat. Methods 2010, 7, 733-736.

[7] Turney, S. G.; Bridgman, P. C. Laminin stimulates and guides axonal outgrowth via growth cone myosin II activity. Nat. Neurosci. 2005, 8, $717-719$.

[8] Millaruelo, A. I.; Nieto-Sampedro, M.; Cotman, C. W. Cooperation between nerve growth factor and laminin or fibronectin in promoting sensory neuron survival and neurite outgrowth. Dev. Brain Res. 1988, 38, 219-228.

[9] Hammarback, J. A.; Palm, S. L.; Furcht, L. T.; Letourneau, P. C. Guidance of neurite outgrowth by pathways of substratum-adsorbed laminin. $J$. Neurosci. Res. 1985, 13, 213-220.

[10] Gundersen, R. W. Response of sensory neurites and growth cones to patterned substrata of laminin and fibronectin in vitro. Dev. Biol. 1987, 121, $423-431$. 
[11] Kostarelos, K.; Bianco, A.; Prato, M. Promises, facts and challenges for carbon nanotubes in imaging and therapeutics. Nat. Nanotechnol. 2009, 4, 627-633.

[12] Zhang, B. B.; Yan, W.; Zhu, Y. J.; Yang, W. T.; Le, W. J.; Chen, B. D.; Zhu, R. R.; Cheng, L. M. Nanomaterials in neural-stem-cell-mediated regenerative medicine: Imaging and treatment of neurological diseases. Adv. Mater. 2018, 30, 1705694.

[13] Besteman, K.; Lee, J. O.; Wiertz, F. G. M.; Heering, H. A.; Dekker, C. Enzyme-coated carbon nanotubes as single-molecule biosensors. Nano Lett. 2003, 3, 727-730.

[14] Li, X. M.; Liu, H. F.; Niu, X. F.; Yu, B.; Fan, Y. B.; Feng, Q. L.; Cui, F. Z.; Watari, F. The use of carbon nanotubes to induce osteogenic differentiation of human adipose-derived MSCs in vitro and ectopic bone formation in vivo. Biomaterials 2012, 33, 4818-4827.

[15] Béduer, A.; Seichepine, F.; Flahaut, E.; Loubinoux, I.; Vaysse, L.; Vieu, C. Elucidation of the role of carbon nanotube patterns on the development of cultured neuronal cells. Langmuir 2012, 28, 17363-17371.

[16] Chao, T. I.; Xiang, S. H.; Lipstate, J. F.; Wang, C. C.; Lu, J. Poly(methacrylic acid)-grafted carbon nanotube scaffolds enhance differentiation of hESCs into neuronal cells. Adv. Mater. 2010, 22, 3542-3547.

[17] Fabbro, A.; Villari, A.; Laishram, J.; Scaini, D.; Toma, F. M.; Turco, A.; Prato, M.; Ballerini, L. Spinal cord explants use carbon nanotube interfaces to enhance neurite outgrowth and to fortify synaptic inputs. ACS Nano 2012, 6, 2041-2055.

[18] Shin, S. R.; Jung, S. M.; Zalabany, M.; Kim, K.; Zorlutuna, P.; Kim, S. B.; Nikkhah, M.; Khabiry, M.; Azize, M.; Kong, J. et al. Carbon-nanotubeembedded hydrogel sheets for engineering cardiac constructs and bioactuators. ACS Nano 2013, 7, 2369-2380.

[19] Shin, S. R.; Bae, H.; Cha, J. M.; Mun, J. Y.; Chen, Y. C.; Tekin, H.; Shin, H.; Farshchi, S.; Dokmeci, M. R.; Tang, S. et al. Carbon nanotube reinforced hybrid microgels as scaffold materials for cell encapsulation. ACS Nano 2012, 6, 362-372.

[20] Xie, X.; Zhao, W. T.; Lee, H. R.; Liu, C.; Ye, M.; Xie, W. J.; Cui, B. X.; Criddle, C. S.; Cui, Y. Enhancing the nanomaterial bio-interface by addition of mesoscale secondary features: Crinkling of carbon nanotube films to create subcellular ridges. ACS Nano 2014, 8, 11958-11965.

[21] Cellot, G.; Cilia, E.; Cipollone, S.; Rancic, V.; Sucapane, A.; Giordani, S.;
Gambazzi, L.; Markram, H.; Grandolfo, M.; Scaini, D. et al. Carbon nanotubes might improve neuronal performance by favouring electrical shortcuts. Nat. Nanotechnol. 2009, 4, 126-133.

[22] Gheith, M. K.; Pappas, T. C.; Liopo, A. V.; Sinani, V. A.; Shim, B. S.; Motamedi, M.; Wicksted, J. P.; Kotov, N. A. Stimulation of neural cells by lateral currents in conductive layer-by-layer films of single-walled carbon nanotubes. Adv. Mater. 2006, 18, 2975-2979.

[23] Wu, C. H.; Liu, A. M.; Chen, S. P.; Zhang, X. F.; Chen, L.; Zhu, Y. D.; Xiao, Z. W.; Sun, J.; Luo, H. R.; Fan, H. S. Cell-laden electroconductive hydrogel simulating nerve matrix to deliver electrical cues and promote neurogenesis. ACS Appl. Mater. Interfaces 2019, 11, 22152-22163.

[24] Barrejón, M.; Rauti, R.; Ballerini, L.; Prato, M. Chemically cross-linked carbon nanotube films engineered to control neuronal signaling. ACS Nano 2019, 13, 8879-8889.

[25] Zhang, X.; Prasad, S.; Niyogi, S.; Morgan, A.; Ozkan, M.; Ozkan, C. S. Guided neurite growth on patterned carbon nanotubes. Sens. Actuators B Chem. 2005, 106, 843-850.

[26] Suzuki, I. K.; Vanderhaeghen, P. Is this a brain which I see before me? Modeling human neural development with pluripotent stem cells. Development 2015, 142, 3138-3150

[27] Avior, Y.; Sagi, I.; Benvenisty, N. Pluripotent stem cells in disease modelling and drug discovery. Nat. Rev. Mol. Cell Biol. 2016, 17, 170-182.

[28] Kasteel, E. E. J.; Westerink, R. H. S. Comparison of the acute inhibitory effects of tetrodotoxin (TTX) in rat and human neuronal networks for risk assessment purposes. Toxicol. Lett. 2017, 270, 12-16.

[29] Lovat, V.; Pantarotto, D.; Lagostena, L.; Cacciari, B.; Grandolfo, M.; Righi, M.; Spalluto, G.; Prato, M.; Ballerini, L. Carbon nanotube substrates boost neuronal electrical signaling. Nano Lett. 2005, 5, 1107-1110.

[30] Discher, D. E.; Janmey, P.; Wang, Y. L. Tissue cells feel and respond to the stiffness of their substrate. Science 2005, 310, 1139-1143.

[31] Ali, S.; Wall, I. B.; Mason, C.; Pelling, A. E.; Veraitch, F. S. The effect of Young's modulus on the neuronal differentiation of mouse embryonic stem cells. Acta Biomater. 2015, 25, 253-267.

[32] Young, A.; Machacek, D. W.; Dhara, S. K.; MacLeish, P. R.; Benveniste, M.; Dodla, M. C.; Sturkie, C. D.; Stice, S. L. Ion channels and ionotropic receptors in human embryonic stem cell derived neural progenitors. Neuroscience 2011, 192, 793-805. 